PROCEEDINGS OF THE

AMERICAN MATHEMATICAL SOCIETY

Volume 45, Number 1, July 1974

\title{
COALGEBRAIC COALGEBRAS
}

\section{E. RADFORD}

ABSTRACT. We investigate coalgebras $C$ over a field $k$ such that the dual algebra $C^{*}$ is an algebraic algebra ( $C$ is called coalgebraic). The study reduces to the cosemisimple and connected cases. If $C$ is cosemisimple and coalgebraic, then $C^{*}$ is of bounded degree. If $C$ is connected, then $C$ is coalgebraic if, and only if, every coideal is the intersection of cofinite coideals. Our main result is that if $C$ is a coalgebra over an infinite field $k$ and the Jacobson radical $\operatorname{Rad} C^{*}$ is nil, there is an $n$ such that $a^{n}=0$ all $a \in \operatorname{Rad} C^{*}$. By the Nagata-Higman theorem, $\operatorname{Rad} C^{*}$ is nilpotent if nil in characteristic 0 .

0. Introduction. In this paper we investigate coalgebras $C$ over a field $k$ such that the dual algebra $C^{*}$ is algebraic (i.e. $k[a]$ is finite dimensional for all $a \in C^{*}$ ). Such coalgebras we call coalgebraic. An ideal $I$ of $A=C^{*}$ is said to be algebraic if $A / I$ is an algebraic algebra. It is apparent that $D \mapsto D \perp$ gives a bijective correspondence of the coalgebraic subcoalgebras of $C$ and the closed algebraic ideals of $C^{*}$. Applications to algebraic ideals will be a minor theme in this discussion.

A basic observation one makes is that the study of coalgebraic coalgebras reduces to the cosemisimple and connected cases. The connected case is the more difficult and interesting. The Jacobson radical of $C^{*}$ plays a central part in the latter. Our main result is that if $C$ is any coalgebra over an infinite field and $J=\operatorname{Rad} C^{*}$ is nil, then there is an $n$ such that $a^{n}=0$ all $a \in J$. Consequently in characteristic 0 the Jacobson radical of $C^{*}$ is nilpotent if it is nil. Combining our main theorem with a result from the cosemisimple case, we conclude that if $C$ is a coalgebraic coalgebra over an infinite field, then $C^{*}$ is an algebraic algebra of bounded degree. We show that coalgebraic coalgebras over an infinite field which are of finite type must be finite dimensional. As a corollary, for locally finite coalgebras over an infinite field the closed algebraic ideals of $C^{*}$ are cofinite.

Received by the editors October 16, 1972 and, in revised form, June 20, 1973. AMS (MOS) subject classifications (1970). Primary 16A38, 16A21, 16A22; Secondary $16 A 24$.

Key words and phrases. Algebraic algebra, Jacobson radical, dual algebra of a coalge bra. 
1. Preliminaries. We shall denote a coalgebra $(C, \Delta, \epsilon)$ simply as $C$. Throughout this paper the dual algebra $C^{*}$ will be regarded as a topological space with the weak-* topology. For a subspace $V$ of $C$ let $V^{\perp}=\left\{a \in C^{*}\right.$ : $a(v)=0$ all $v \in V\}$ and for a subspace $I$ of $C^{*}$ let $I^{\perp}=\{c \in C: a(c)=0$ all $a \in I\}$. Letting $I$ denote the closure of a subspace $I$ of $C^{*}$, one should notice that $I=I^{\perp \perp}$. If $I$ is a subspace of $C^{*}$ we say a subspace $J$ contained in $I$ is dense (in $I$ ) if $\bar{J}=\bar{I}$. Notice that a linear map $f: C \rightarrow D$ induces a continuous map $f^{*}: D^{*} \rightarrow C^{*}$.

Let $J=\operatorname{Rad} C^{*}$ be the Jacobson radical of the dual algebra $C^{*}$ of a coalgebra $C$. The powers of $J$ induce a filtration on $C$ where $C_{n}=\left(J^{n+1}\right) \perp$. In particular $C_{0}=J^{\perp}$ is the sum of the simple subcoalgebras of $C$ (see [5]). This implies $J \subset C_{0}^{1}$. The other inclusion holds also.

\subsection{Lemma (2.1.4 of [2]). If $C$ is a coalgebra, then $C_{0}^{1}=\operatorname{Rad} C^{*}$.}

Proof. It suffices to show $C_{0}^{1} \subset \operatorname{Rad} C^{*}$. Suppose $a \in C_{0}^{1}$. Then $u=$ $\sum_{n=0}^{\infty} a^{n}$ is well defined, and one checks that $u^{*}(1-a)=1=(1-a)^{*} u$. Since $C_{0}^{1}$ is an ideal, we are done. Q.E.D.

For a coalgebra $C$ let $C^{+}=\operatorname{ker} \epsilon$, and define $R(C)=C / C_{0}^{+}$. Then $R(C)$ is connected, and by 1.1

1.2. $R(C)^{*} \simeq \operatorname{Rad} C^{*} \oplus k \cdot 1$.

Since subalgebras and quotients of algebraic algebras are algebraic, quotients and subcoalgebras of coalgebraic coalgebras are coalgebraic. The next lemma provides a useful converse.

1.3 Lemma. Suppose $D$ is a subcoalgebra of a coalgebra $C$. Then $C$ is coalgebraic if $D$ and $C / D^{+}$are.

Proof. Suppose $D$ and $E=C / D^{+}$are coalgebraic. If $I=D^{\perp}$, then $0 \rightarrow$ $I \rightarrow C^{*} \rightarrow{ }^{u} D^{*} \rightarrow 0$ is exact ( $u$ the restriction map) and $E^{*} \simeq I \oplus k$. 1 . Thus $k[b]$ is finite dimensional for $b \in I$. Now let $a \in C^{*}$. Then $k[u(a)]$ is finite dimensional, so

$$
0=a_{0} 1+\cdots+u(a)^{n}=u\left(a_{0} 1+\cdots+a^{n}\right) \text { for some } n>0 \text { and } a_{i} \in k .
$$

Thus $b=\alpha_{0} 1+\cdots+a^{n} \in I$. Since $k[b]$ is finite dimensional we have $0=$ $\beta_{0} 1+\cdots+a^{n m}$ for some $m>0$ and $\beta_{i} \in k$. Q.E.D.

1.4 Corollary. A coalgebra $C$ is coalgebraic if, and only if, $C_{0}$ and $R(C)$ are. 
By virtue of the preceding corollary the study of coalgebraic coalgebras is reduced to the cosemisimple and connected cases.

If $C=C_{n}$ then $\operatorname{Rad} C^{*}$ is nilpotent. As a corollary to 1.4 we have:

1.5 Corollary. Suppose $C$ is a coalgebra. If $C_{0}$ is coalgebraic then $C_{n}$ is all $n$.

2. The cosemisimple case. This case is by far the simpler, and one can relatively easily give a complete a nalysis. The group-like coalgebra $k^{(X)}$ of a set $X$ over a field $k$ will play an important role. Recall that this coalgebra is the $k$-vector space with basis $X$ with comultiplication deter-

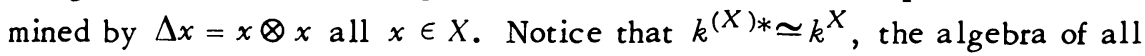
functions $f: X \rightarrow k$ under pointwise multiplication. Observe that:

2.1. If $k$ is infinite, then $k^{(X)}$ is coalgebraic only if $X$ is finite.

The proof is trivial. For if $f \in k^{X}$ and $p(f)=0$ for some $0 \neq p(X) \epsilon$ $k[X]$, then $p(f(x))=0$ all $x \in X$ which implies $f(x)$ assumes only finitely many values.

2.1 is in sharp consrast to the finite case; if $k$ is finite then $k^{(X)}$ is coalgebraic for all sets $X$.

2.2. Proposition. Suppose $C$ is cosemisimple and coalgebraic.

(1) If $k$ is infinite, then $C$ is finite dimensional.

(2) If $k$ is finite, there exists an $n>0$ such that $\operatorname{dim} D \leq n$ for all simple subcoalgebras $D$ of $C$.

Proof. Write $C=\amalg\left\{C_{x}: x \in X\right\}$ where each $C_{x}$ is simple. Then $k^{X}$ is a subalgebra of $C^{*}$.

Suppose $k$ is infinite. Now $k^{X}$ is algebraic since $C^{*}$ is. By $2.1 X$ is finite:

Suppose $k$ is finite. Then we may essentially repeat the argument for 2.1 (replace $k^{(X)}$ by $\amalg K_{x}^{*}$ where $K_{x}$ is an appropriate field extension of $k$ ) once we observe: if $A$ is a simple algebra over a finite field $k$ and $\operatorname{dim} A=n$, there is a subalgebra $K$ which is a field extension of $k$ such that $[K: k] \geq n^{1 / 2}$. Q.E.D.

It is clear that the necessary conditions in the preceding proposition are also sufficient.

2.3 Corollary. If $C$ is cosemisimple and coalgebraic, then $C^{*}$ is an algebraic algebra of bounded degree (hence satisfies a polynomial identity). 
If $k$ is finite, the proof of 2.3 is clear; if $k$ is infinite we appeal to 2.34 of $[1]$ in addition to 2.2 .

For an ideal $I$ of an algebra $A$ we shall for convenience denote the intersection of all the maximal ideals containing $I$ by $\operatorname{Rad}(I)$. The following observation is useful.

2.4. If $I$ is a closed ideal of $A=C^{*}$ then $\operatorname{Rad}(I)$ is closed.

Proof. If $D=I^{\perp}$ we have the exact sequence $0 \rightarrow I \rightarrow C^{*} \rightarrow^{u} D^{*} \rightarrow 0$ where $u$ is the restriction map. It is clear that $\operatorname{Rad}(I)=u^{-1}\left(\operatorname{Rad} D^{*}\right)$. This means $\operatorname{Rad}(I)$ is closed since $\operatorname{Rad} D^{*}$ is. Q.E.D.

An immediate consequence of 2.2 and 2.4 is:

2.5 Corollary. Suppose $C$ is a coalgebra over an infinite field. If I is a closed algebraic ideal of $C^{*}$ then $\operatorname{Rad}(I)$ is closed and cofinite.

Recall that any subcoalgebra of $k^{(X)}$ has the form $k^{(Y)}$ for some subset $Y$ of $X$. This means any closed ideal of $k^{X}$ has the form $I(Y)=\left\{f \in k^{X}\right.$ : $f(Y)=(0)\}$. Notice we have the exact sequence $0 \rightarrow I(Y) \rightarrow k^{X} \rightarrow k^{Y} \rightarrow 0$. By 2.1

2.6. If $k$ is infinite and $I$ is an algebraic ideal of $k^{X}$, then the smallest closed ideal containing $I$ is cofinite.

Now suppose $k$ is any field and $X$ any set. One can easily prove that the algebraic maximal ideals of $k^{X}$ have codimension 1 . Suppose $I$ is any ideal of $k^{X}$. Then $I$ is the intersection of prime ideals. Since algebraic prime ideals are maximal in commutative algebras, we conclude that any algebraic ideal of $k^{X}$ is the intersection of maximal ideals of codimension 1 .

A coalgebra $C$ is called coreflexive if the cofinite ideals of $C^{*}$ are closed (see [4]).

2.7 Proposition. Let $C$ be cosemisimple and coreflexive. Then the algebraic ideals of $C^{*}$ are cofinite (hence closed).

Proof. Write $C=\amalg\left\{C_{x}: x \in X\right\}$ where $C_{x}$ is a simple subcoalgebra, and for each subset $N$ of positive integers set $C[N]=\amalg\left\{C_{x}: \operatorname{dim} C_{x} \in N\right\}$. Let $I$ be any algebraic ideal of $C^{*}$. Then by 3.9 of [4], there is a finite $N$ such that $I=u^{-1}(u(I))$, where $C^{*} \rightarrow^{u} C[N]^{*}$ is the restriction.

Case 1. $k$ finite. $C[N]$ is finite by 3.12 of [4].

Case 2. $k$ infinite. For some subset $Y$ of $X$ we have $C[N]=\amalg\left\{C_{x}\right.$ : $x \in Y\}$. Now $k^{Y}$ is a subalgebra of $A=C[N]^{*}$, and furthermore $A$ is a finitely generated left $k^{Y}$ module.

Now $J=u(I)$ is an algebraic ideal of $A$, so $\mathbb{M}=J \cap k^{Y}$ is an algebraic ideal of $k^{Y}$. But $k^{(Y)}$ is coreflexive by 3.12 of [4], so $\mathbb{M}$ is closed and 
cofinite by 2.6 and the preceding remark. Hence by 1.3 .8 of [3], $M \cdot A$ is a cofinite subspace of $A$, and $\mathbb{M} \cdot A \subset J$ implies that $J$ is cofinite. Thus $I=u^{-1}(J)$ is cofinite. Q.E.D.

We conclude this section with two immediate corollaries.

2.8 Corollary. If $C_{0}$ is coreflexive the algebraic ideals which contain $\operatorname{Rad} C^{*}$ are cofinite and closed.

2.9 Corollary. If $C_{0}$ is coreflexive, then $\operatorname{Rad}(I)$ is cofinite and closed whenever $I$ is an algebraic ideal of $C^{*}$.

3. The connected case. In this section we treat the connected case. One should notice that the study of the Jacobson radical $\operatorname{Rad} C^{*}$ of $C^{*}$ and the connected coalgebra $R(C)$ are really the same since $R(C)^{*} \simeq \operatorname{Rad} C^{*} \oplus$ $k \cdot 1$. For any algebra $A$ over a field it is well known that the algebra $\operatorname{Rad} A$ is algebraic if, and only if, it is nil. Thus any connected coalgebra $C$ (or more specifically $R(C)$ ) is coalgebraic if, and only if, $\operatorname{Rad} C^{*}$ is nil. Our first proposition characterizes internally coalgebraic connected coalgebras.

3.1 Proposition. For a connected coalgebra $C$ the following statements are equivalent:

(1) $C$ is coalgebraic.

(2) The span of the finite dimensional subalgebras of any closed subalgebra $B$ of $C^{*}$ is dense (in $B$ ).

(3) Every coideal of $C$ is the intersection of cofinite coideals.

Proof. (1) $\Rightarrow(2)$ Clearly true for any coalgebraic coalgebra.

$(2) \Rightarrow$ (3) Again, clear for any coalgebra since $\left(\Sigma B_{a}\right)^{\perp}=\bigcap B_{a}^{\perp}$ for subspaces of $C^{*}$.

(3) $\Rightarrow(1)$. If (3) holds for a coalgebra $C$ then it holds for quotients and subcoalgebras as well. So let $a \in \operatorname{Rad} C^{*}$ and $I=k[a]^{\perp}$. Then $D=C / I$ is connected since $C$ is, and $D^{*} \simeq \mathrm{cl}(k[a])$, the closure of $k[a]$. Let $D^{*} \rightarrow^{u} D_{1}^{*}$, be the restriction map. Then $u(k[a])$ is a dense subalgebra of $D_{1}^{*}$. But $u(a)^{2}=0$ which implies that the space of primitives $P(D)=k \cdot v$ for some $v \in D$. Therefore by 12.1 .1 on p. 254 of [5] we have an embedding into the "shuffle algebra" $D \rightarrow \operatorname{Sh}(k \cdot v)$ with $D_{1} \simeq \operatorname{Sh}(k \cdot v)_{1}$. If $D$ is not finite dimensional then $D \simeq$ $\operatorname{Sh}(k \cdot v)$ which implies $D^{*} \simeq k[[X]]$, the power series ring in $X$ over $k$. But $k \cdot 1$ is the only finite dimensional subalgebra of $k[[X]]$. Hence $D$ is finite dimensional, and this means $k[a]$ is also. Therefore $C$ is coalgebraic. Q.E.D. 
The preceding proposition is not true in general since $(3) \not \Rightarrow$ (1) in the cosemisimple case. If $C$ is any cocommutative cosemis imple coalgebra then the intersection of all cofinite coideals is (0). But if $I$ is any coideal of $C$ then $C / I$ is also cosemisimple (the intersection of cofinite maximal ideals of any subalgebra of $C^{*}$ is $(0)$ in the commutative case).

We now come to the main result of this paper.

3.2 Theorem. Suppose $C$ is a coalgebra over an infinite field $k$ and $J=\operatorname{Rad} C^{*}$ is nil. Then $a^{n}=0$ all $a \in J$ for some fixed $n$.

Proof. Suppose there is no such $n$. We will obtain a contradiction by constructing an $a \in J$ such that $a^{m} \neq 0$ all $m>0$. To do this it will be sufficient to find a sequence $a_{1}, a_{2}, \cdots \in J$ and an increasing sequence of integers $0=f(0)<f(1)<f(2) \cdots$ such that

(1) $a_{n}\left(C_{f(n-1)}\right)=(0)$, and

(2) $\Sigma_{\left(i_{1}, \cdots, i_{n}\right) ; 1 \leq i_{j} \leq n} a_{i_{1}} \cdots a_{i_{n}} \equiv 0$ on $C_{f(n)}$ for all $n \geq 1$.

Since the $C_{n}$ 's form an increasing filtration $a=\sum_{n=1}^{\infty} a_{n}$ is well defined, and for $m>0$

$$
a^{m}=\sum_{\left(i_{1}, \cdots, i_{m}\right)} a_{i_{1}} \cdots \cdots a_{i_{m}} \equiv \sum_{\left(i_{1}, \cdots, i_{m}\right) ; 1 \leq i_{j} \leq m} a_{i_{1}} \cdots \cdots a_{i_{m}} \not \equiv 0
$$

when restricted to $C_{f(m)}$. Thus $a^{m} \neq 0$ all $m>0$.

We will find these sequences simultaneously by induction. $J \neq(0)$ by assumption, so choose $0 \neq a_{1} \in J$. Now $a_{1}\left(C_{r}\right) \neq(0)$ for some $r$, so let $f(1)$ be such an $r$. Assume $a_{1}, \cdots, a_{n}$ and $f(1), \cdots, f(n)$ have been chosen by induction. For each $0 \leq l \leq n+1$ we define a function $g_{l}: J \rightarrow J$ as follows: let $b_{i}=a_{i}$ for $1 \leq i \leq n$ and for $b \in J$ let $b_{n+1}=b$; finally set

$$
g_{l}(b)=\sum_{\left(i_{1}, \cdots, i_{n+1}\right) ; 1 \leq i_{j} \leq n+1}^{\prime} b_{i_{1}} \cdot \ldots \cdot b_{i_{n+1}}
$$

where exactly $l$ of $i_{1}, \cdots, i_{n+1}$ are $n+1$. Notice that $g_{l}(a b)=\alpha^{l} g_{l}(b)$ for all $b \in J$ and $a \in k$.

Let $g(b)=\sum_{l=0}^{n+1} g_{l}(b)$. Suppose $g(b)=0$ for all $b \in J^{f(n)+1}$. Then for such a $b$ and any $c \in C$ we have $0=g(\alpha b)(c)=\sum_{l=0}^{n+1}\left(g_{l}(b)(c)\right) \alpha^{l}$ for all $a \in k$. Since $k$ is infinite, $g_{l}(b)(c)=0$ all $0 \leq l \leq n+1$, so $0=g_{n+1}(b)=$ $b^{n+1}$ in particular. This implies $a^{(f(n)+1)(n+1)}=0$ for all $a \in J$, a contradiction. Therefore $g\left(b_{0}\right) \neq 0$ for some $b_{0} \in J^{f(n)+1}$. Set $a_{n+1}=b_{0}$. Then 
$a_{n+1}\left(C_{f(n)}\right)=(0)$, and since $g\left(a_{n+1}\right) \neq 0$ we have $g\left(a_{n+1}\right)\left(C_{r}\right) \neq(0)$ for some $r$. Let $f(n+1)=\max \{r, f(n)+1\}$. Q.E.D.

Combining 3.2 with 2.3:

3.3 Corollary. If $C$ is a coalgebraic coalgebra over an infinite field then $C^{*}$ is an algebraic algebra of bounded degree (hence satisfies a polynomial identity).

The Nagata-Higman theorem $[1,1.13]$ together with 3.2 gives the following result in characteristic 0 .

3.4 Proposition. Suppose $C$ is a coalgebra over a field of characteris tic 0. Then $\operatorname{Rad} C^{*}$ is nilpotent if it is nil.

In what follows a ring $R$ does not necessarily have a unit element. Recall that a ring $R$ is called locally nilpotent if every finitely generated subring is nilpotent. We quote an important theorem of ring theory $[1,1.15]$.

Theorem (Levitzki). Let $R$ be a ring in which $x^{n}=0$ all $x \in R$ for some fixed $n$. Then $R$ is locally nilpotent.

An immediate consequence of Levitzki's theorem and 3.2 is:

3.5 Corollary. Rad $C^{*}$ is locally nilpotent if it is nil and the ground field is infinite.

It is well known that an algebraic algebra satisfying a polynomial identity is locally finite. However we prefer to give a more basic proof of the following proposition using coalgebra techniques.

3.6 Proposition. Suppose $C$ is a coalgebra over an infinite field and $\operatorname{Rad} C^{*}$ is nil. Then $\operatorname{Rad} C^{*}$ is finite dimensional if it contains a dense finitely generated subring.

Proof. We may assume that $C=R(C)$. So suppose $C$ is connected and $R$ is a finitely generated subring dense in $\operatorname{Rad} C^{*}$. The linear span $V$ of $R$ is also dense in $\operatorname{Rad} C^{*}$. By $3.5 R^{m}=(0)$ some $m$ so clearly $V^{m}=(0)$ also. Now $V$ is a finitely generated left ideal of the algebra $A=V \oplus k \cdot 1$. Using 1.3.8 of [3] we conclude by induction that $V^{n}$ has finite codimension in $V$ for all $n$. Thus $V$ is finite dimensional, and since finite dimensional subspaces are closed, $V=\operatorname{Rad} C^{*}$. Q.E.D.

One should notice that only the hypothesis of Levitzki's theorem was needed in the above argument; in particular $k$ could be finite. 
Remark. It should be noticed that $\operatorname{Rad} C^{*}$ can be replaced in 3.5 and 3.6 by $\bar{R}$ where $R$ is a subring of $\operatorname{Rad} C^{*}$. For $A=\bar{R} \oplus k \cdot 1$ is a closed subalgebra of $C^{*}$. Thus $A \simeq D^{*}$ where $D$ is a quotient of $C$. $D$ must be connected, and therefore $\bar{R} \simeq \operatorname{Rad} D^{*}$.

A coalgebra $C$ is said to be of finite type if $C_{1}$ is finite dimensional.

3.7 Proposition. Suppose $C$ is a coalgebra of finite type over an infinite field $k$. If $C$ is coalgebraic then $C$ is finite dimensional.

Proof. Since $C$ is of finite type and coalgebraic, the same is true of $R(C)$. Thus we may additionally assume that $C$ is connected. By 3.6 we need only find a finitely generated subring dense in $\operatorname{Rad} C^{*}$. But the space of primitives $V=P(C)$ is finite dimensional. By 12.1 .1 on p. 254 of [5] we have an embedding $C \rightarrow \operatorname{Sh}(V)$. But $\mathrm{Sh}(V)^{*}$ can be identified with the power series ring in $n=\operatorname{dim} V$ noncommuting indeterminants $X_{1}, \cdots, X_{n}$ over $k$. The desired subring is generated by the ir images under the surjective composition $k\left\{\left\{X_{1}, \cdots, X_{n}\right\}\right\} \simeq \mathrm{Sh}(V)^{*} \rightarrow C^{*}$. Q.E.D.

Using the definition of " $\wedge$ " found on p. 179 of [5] we call a coalgebra $C$ locally finite if $D \wedge D$ is finite dimensional for all finite dimensional subcoalgebras $D$ of $C$.

3.8 Corollary. Suppose $C$ is a locally finite coalgebra over an infinite field $k$. Then the closed algebraic ideals of $C^{*}$ are cofinite.

Proof. Let $I$ be a closed algebraic ideal of $C^{*}$ and $D=I^{\perp}$. Then $D$ is coalgebraic so $D_{0}$ is finite dimensional by 2.2. By ass umption $D$ is of finite type. By 3.7, D is finite dimensional. Therefore $I=D^{\perp}$ is cofinite. Q.E.D.

\section{REFERENCES}

1. I. N. Herste in, Theory of rings, Math. Lecture Note Series, University of Chicago, Chicago, Ill., 1961.

2. R. G. Heyneman and D. E. Radford, Reflexivity and coalgebras of finite type, J. Algebra 28 (1974), 215-246.

3. R. G. Heyneman and M. E. Sweedler, Affine Hopf algebras. I, J. Algebra 13 (1969), 192-241. MR 39 \#6876.

4. D. E. Radford, Coreflexive coalgebras, J. Algebra 26 (1973), 512-535.

5. M. E. Sweedler, Hopf algebras, Math. Lecture Note Series, Benjamin, New York, 1969. MR 40 \#705.

DEPARTMENT OF MATHEMATICS, LAWRENCE UNIVERSITY, APPLETON, WISCONSIN 54911 